\title{
A comment on multidimensional poverty indices
}

\author{
Erik Thorbecke
}

Published online: 2 August 2011

(c) Springer Science+Business Media, LLC. 2011

The three papers on multidimensional poverty published in the 2011 June issue of the Journal of Economic Inequality [1-3] present strong cases in favor of their respective methodologies to capture and measure well-being and poverty. Ultimately the choice of preferred indicators, whether unidimensional or multidimensional (and, in the latter case, which dimensions to incorporate in such indicators), depends ultimately on the purposes they should serve. Money-metric (income or consumption based) poverty measures have been useful to the World Bank in estimating approximately the magnitude of global poverty as well as individual regions' and countries' progress towards the reduction of poverty. The Human Development Index has provided the development community with a broader measure of well-being-adding health and educational dimensions to income-and demonstrating that the correlation between the monetary and non-monetary dimensions of poverty within and across countries was far from perfect. Finally, multidimensional indicators such as the Alkire-Foster (A-F) measure respond to an effective demand for scalar estimates of poverty that could be used by policymakers in the allocation of funds to reduce poverty in an efficient and equitable way. Hence, notwithstanding their shortcomings, each of these indicators fulfills somewhat different yet important functions.

A strong feature of the A-F methodology is that it is anchored on the joint distribution of the various dimensions of well-being and the resulting deprivations that poor people experience. It is therefore essential that all data come from the same household survey. It should be noted that this means that any given household information is available not only on possible deprivations in some dimensions but also on possible surpluses over and above the deprivation thresholds in other dimensions. This immediately raises the issue of possible trade-offs among dimensions.

E. Thorbecke $(\bowtie)$

Cornell University, Ithaca, NY, USA

e-mail: et17@cornell.edu 
For example, a household classified as deprived in terms of nutrition because its food consumption is below the recommended dietary caloric threshold but with an income above the income poverty line could, in principle, compensate for the food shortage by drawing on its income surplus to improve its nutrition. To emphasize and illustrate this point, assume two households with the same demographic pattern. Household $\mathrm{A}$ is $20 \%$ below the nutrition cut-off and at the income poverty level while household B is $20 \%$ below the nutrition cut-off but $20 \%$ above the income poverty line. I would venture to guess that most analysts would consider B to be better off than A and less poor. The A-F measure would treat these two households similarly because it rules out substitutability or complementarity between pairs of dimensions. Essentially, Alkire and Foster [1] ignore all household information above the deprivation thresholds for those households that are not deprived in that dimension. For any given household deemed poor in one or more dimensions, it is important to know how it fares in terms of the dimensions in which it is not deprived.

While it appears to make sense for unidimensional poverty measures to ignore the income distribution of the non-poor, it is a very different proposition to ignore the well-being profile of households who are poor in only some dimensions. I am sympathetic with the argument that in a multidimensional setting the interrelationships among all dimensions under consideration are so complex that from an operational standpoint it would be an almost hopeless task to estimate all the tradeoffs and complementary relations between pairs of dimensions. Yet this issue is so conceptually important that it cannot be rationalized away.

A second issue relates to the arbitrariness of the various decisions required in constructing a multidimensional poverty measure, i.e. the selection of the dimensions, dimensional thresholds, dimensional weights and a poverty cut-off in the A-F framework. Given all these contingent decisions, it would be interesting and revealing to explore the sensitivity of the results in terms of who is poor or not to different sets and combinations of decisions regarding choice of dimensions, thresholds, relative weights and poverty cut-offs. Of course, a potential advantage of the A-F approach is the flexibility it provides to the users in the choice of the above decisions. Yet, I would speculate that policymakers and analysts would benefit from such sensitivity analysis and that it would improve the quality of their decisions.

Part of the flexibility inherent in the A-F methodology follows from the choice of relative dimensional weights. In theory, this procedure provides an opportunity for analysts to alter the implicit market prices reflected in the consumption baskets chosen by the various households. An appropriate choice of weights could act like accounting or shadow prices to substitute for missing markets and market prices not reflecting the marginal social utilities embraced by the analysts/users. However the downside of this procedure is that it would conflict with welfare consistency as Ravallion [3] emphasizes.

Finally, I have long advocated the use of poverty profiles. As a second best alternative to multidimensional poverty indicators one could build individual profiles of normalized wellbeing in each of the dimensions. Each point on a given profile for a given individual could be interpreted as representing a relative shortfall below the dimensional threshold (showing a deprivation) or a surplus over and above the threshold for other dimensions. Such individual profiles would need to be aggregated to yield the type of information useful to policymakers-a very difficult task as the three papers made abundantly clear. 


\section{References}

1. Alkire, S., Foster, J.: Understandings and misunderstandings of multidimensional poverty measurement. J. Econ. Inequal. 9(2), 289-314 (2011)

2. Klugman, J., Rodríguez, F., Choi, H.-J.: The HDI 2010: new controversies, old critiques. J. Econ. Inequal. 9(2), (2011)

3. Ravallion, M.: On multidimensional indices of poverty. J. Econ. Inequal. 9(2), 235-248 (2011) 